International Journal of Electrical Engineering and Technology (IJEET)

Volume 11, Issue 4, June 2020, pp. 331-340, Article ID: IJEET_11_04_037

Available online at https://iaeme.com/Home/issue/IJEET? Volume $=11 \&$ Issue $=4$

ISSN Print: 0976-6545 and ISSN Online: 0976-6553

DOI: https://doi.org/10.34218/IJEET.11.4.2020.037

\title{
SOLVING WIND INTEGRATED DYNAMIC ECONOMIC LOAD DISPATCH USING DYNAMIC NEIGHBORHOOD BASED DIFFERENTIAL EVOLUTION
}

\author{
B. Padmanabhan \\ Research Scholar, Sathyabama Institute of Science and Technology, \\ Chennai, Tamilnadu, India \\ L. Premalatha \\ Professor, Vellore Institute of Technology, Chennai, Tamilnadu, India
}

\begin{abstract}
Economically satisfying the power demand of various consumers in dynamic mode becomes a prime challenge nowadays. Unbalanced load variation makes the power demand and power loss vibrant which leads to the rough load curve. To deal with differential power requirement, dynamic economic (DELD) load dispatch is witnessed as major one in consideration with unauthorized operating zone, economic load dispatch in valve pointed loading, etc. On considering the surplus power demand it is mandatory to find an alternate way to satisfy it economically, hence renewable energy support is crucial. The discontinuous power generation of wind energy with respect to the availability of wind source makes the DELD problem non smooth and non convex in practical. Hence an evolutionary computational technique is prominent to find a better solution. In proposed topology, Neighborhood based Differential Evolution Algorithm has been evaluated for resolving the wind integrated DELD problem including various constraints. To check out values attained from proposed method, 15 unit and 10 unit system test data is optimized for various power demand including the wind support and evaluated with the results from the literature.
\end{abstract}

Key words: Ramp Rate Limit, Wind Integrated DELD, Valve Pointed Loading, Unauthorized Operating Zone.

Cite this Article: B. Padmanabhan and L. Premalatha, Solving Wind Integrated Dynamic Economic Load Dispatch using Dynamic Neighborhood based Differential Evolution. International Journal of Electrical Engineering and Technology, 11(4), 2020, pp. 331-340.

https://iaeme.com/Home/issue/IJEET?Volume=11\&Issue $=4$ 
Solving Wind Integrated Dynamic Economic Load Dispatch using Dynamic Neighborhood based Differential Evolution

\section{INTRODUCTION}

Thermal power plants are the chief contributors of global energy market which accounts for around $60 \%$ of global energy generation. But the operation of thermal power plants is constrained by two major limitations such as growing power demand and increasing fuel cost. Thus operating the thermal power economically as well as meeting the power demand has become an important problem in power system optimization.

The first constraint, increased power demand can be met by increasing the power output from the thermal power plants. But in recent years there is a vast decline in fossil fuel resources and increase in environmental pollution which has made the global energy market to turn its attention towards renewable energy sources. In the year 2018 the renewable energy sources have contributed more for the global power generation. Renewable energy sources offer both economic and environmental benefits. The major renewable resources are wind, solar, tidal, hydro, and geothermal. Of these renewable sources wind and solar are the most common energy sources that have contributed to the huge growth in the global energy market. The wave energy and geothermal energy faces constraints of financing obstacles that is keeping them from commercial success. Also geothermal, biomass contributes a little amount of chemical emission and requires a small amount of water resources when compared to wind and solar based sources which produce pollution free energy. Further wind and solar power has a growing market in global energy market. This necessitates the integration of solar and wind power generation with the conventional thermal power generation. This work has taken the wind power generation alone in integrating with thermal power plant.

The second constraint, economic operation of power plant is solved by optimal scheduling of all the generators in a power plant to meet the forecasted load demand. But the power demand in any system keeps changing with the time. This necessitates the importance of resolve the problem occurred due to dynamic economic load dispatch (DELD) to obtain a real time optimal scheduling of committed units in a power plant[2] [1]. In practical the in and out of production unit tends to be non-convex (or) gradually increasing. The valve point effect due to multiple steam admission valves of turbines and ramp rate limits causes discontinuities in the input-output curve. Further due to the physical vibrations in the shaft, the thermal units have certain prohibited operating zones [12]. To replicate an exact practical system, the above constraints are included in modeling a DELD problem. In addition to the system constraints the DELD with integrated wind energy introduces many local minima with in the search space which makes the WDELD, the most difficult problem.

Few years ago Lambda iteration method, quadratic programming, Newton's method and Lagrangian approach were applied in solving the economic dispatch problem. But these methods faced limitations when the assumed convex fuel cost functions were replaced with real time non convex ones. Though dynamic and non linear programming became a replacement for conventional methods they still suffered from the problem of dimensionality with increasing number of generators [13] [14]. This led researchers to focus in developing new algorithms with global searching abilities. GE called as genetic algorithm, PSO named as Particle swarm optimization, Simulated Annealing, Differential evolution, artificial immune system, and harmony search algorithm are few such nature inspired algorithms that has played major role in solving non linear DELD problems. But still these algorithms face the problems like high computational time, premature convergence, accuracy when the complexity of the objective problem increases [5]. To overcome this, Meta heuristic algorithms were combined with other Meta heuristic algorithms or conventional mathematical methods to develop into a hybrid model. These hybridized models are capable of solving problems involving vagueness, uncertainties and high dimensionalities [4]. 
Many hybrid models like EP-SQP, PSO-SQP have been successfully solved the DELD problem. The hybrid models use a base level search and fine tuner to reach the peerless solution to the given problem. Even though parameter selection and slower convergence is the main drawbacks faced by these hybrid models in solving the WDELD problem. Thus due to the increasing challenges, developing and improving a new hybrid model for the WDELD problem has gained more attention in recent years. This paper focuses in developing one such hybrid model using Differential Evolution.

The differential evolution has been considered as population based evolutionary algorithm and it is chosen as major one in rectifying problems that lack precision and are highly volatile [6]. Thus many articles have been published using DE in solving many real-world applications [7]. Even though DE has outperformed its counterparts, it still has few shortcomings like inability to handle more constraints, weak local exploitation and poor converging velocity [8]. Also with problems like WDELD which has high dimensionality, it suffers with premature convergence, stagnation, parameter handling and loss of diversity [9], [10], and [11]. The DE, competition arises between one by one with relative parent; where differ from other algorithms. This arise faster convergence rate [19]; leads to higher probability to attain local optimum. The reason behind it would be population decreases during solution finding.

To overcome this, DE is presented with dynamic neighborhood [15] which focuses on reducing the computational expenses and increasing the precision by providing a proper tradeoff between the exploration \& exploitation process of DE. The effectiveness of recommended methodology is substantiated for 10 unit and 15 unit system is studied with predecessor. In addition to the usual equality and inequality constraints the valve point effect, ramp rate limit, unauthorized operating zone are considered in solving WDELD problem using proposed methodology.

Through following sections the proposed topology is demonstrated. The next stage reviews the complication identification of WDELD with its constraints. Then the proposed DE is visualized. Empirical and comparison results with conclusion are described in final one.

\section{WIND INTEGRATED DYNAMIC ECONOMIC LOAD DISPATCH}

Dynamic Economic Load Dispatch is enabling to partition online generator's output with the estimated requirement considering major constraints over a cycle.

The objective function be,

$$
F_{T}=\sum_{h=1}^{H} \sum_{i=1}^{N} F_{i}\left(P_{i}\right) \quad \$
$$

Where,

$$
F_{i}\left(P_{i}\right)=a_{i} P_{i}^{2}+b_{i} P_{i}+c_{i} \quad \$ / h r
$$

Where,

$a_{i}, b_{i}, c_{i}-$ coefficient of fuel cost involving $i^{\text {th }}$ unit

$\mathrm{H}-\mathrm{Number}$ of intervals in a day

$\mathrm{N}-\mathrm{N}$ generating units in total

$F_{i}\left(P_{i}\right)$ - Total fuel cost 


\subsection{Power Balance Constraint}

The affixing of the power produced in online thermal units requisite satisfies the power demand including the losses [20]. To incorporate distributed generation in satisfying the power demand, renewable energy support is essential. Hence the wind power support is considered along with Dynamic Power dispatch in this work.

$$
\sum_{i=1}^{N} P_{i}=P_{D}+P_{\text {loss }}-P_{W} \quad M W
$$

Where,

$\mathrm{P}_{\mathrm{D}}-$ Total power demand

$\mathrm{P}_{\mathrm{L}}$ - Total network losses

$\mathrm{P}_{\mathrm{W}}-$ Wind Power Support

\subsection{Capacity Limits Constraints}

Each thermal power unit has its own operating limits on its capacity which varies from minimum to maximum as mentioned below,

$$
P_{i}^{\min } \leq P_{i} \leq P_{i}^{\max } \quad \text { For } \mathrm{i}=1 \ldots . \mathrm{N}
$$

Where,

$\mathrm{P}^{\mathrm{min}}$ - minimal power generation

$\mathrm{P}^{\max }$ - maximized power generation

\subsection{Rectified Sinusoidal Effect}

When the optimization of economic power dispatch problem is concern in theoretical aspects the generation of thermal unit is assumed to be instantaneous and convex. But the operation of thermal units in real time is non smooth \& non convex because of the involvement of sudden change in load which thereby incorporating the sinusoidal quantity in addition to normal fuel cost equation which has no differential points.

$$
F_{i}^{*}\left(P_{i}\right)=F_{i}\left(P_{i}\right)+e_{i} \sin \left(f_{i}\left[P_{i}^{\min }-P_{i}\right]\right) \$ / h r
$$

Where,

$\mathrm{F}_{\mathrm{i}} *\left(\mathrm{P}_{\mathrm{i}}\right)$-fuel cost if $\mathrm{i}^{\mathrm{th}}$ unit regarding valve point effect

$e_{i}, f_{i}$-coefficients of fuel cost having $i^{\text {th }}$ unit regarding valve point effect

\subsection{Ramp Rate Limits}

In reality, thermal units are provided with the limits corresponds to the normal inequality constraint. Hence the thermal units are operated between the specific operating limits continually

As generation increases,

$$
P_{i}-P_{i 0}<=U R_{i}
$$

As generation decreases,

$$
P_{i}-P_{i 0}<=D R_{i}
$$

Otherwise we can written as, 


$$
\begin{aligned}
& \max \left(P_{i}^{\min }, P_{i 0}-D R_{i}\right)<=P_{i} \\
& P_{i}<=\min \left(P_{i}^{\max }, P_{i 0}+U R_{i}\right)
\end{aligned}
$$

Where,

$P_{i}-$ Power Out of $i^{\text {th }}$ unit

$\mathrm{P}_{\mathrm{i} 0}-$ Power output in the former interval of $\mathrm{i}^{\mathrm{th}}$ unit

$\mathrm{UR}_{\mathrm{i}}-\mathrm{i}^{\text {th }}$ unit limit folded with $\mathrm{Up}$-ramp rate limit

$\mathrm{DR}_{\mathrm{i}}-\mathrm{i}^{\text {th }}$ unit folded with Down-ramp rate limit

\subsection{Prohibited Operating Zones}

Depends on the previous operation record of the thermal units, there are certain points identified as non operable point due to non economic operation. Hence, within the operating limits certain spots are identified as prohibited operating zones to fulfill the need economically.

In accordance with POZ the operation can be represented by following equation:

$$
\left\{\begin{array}{c}
P_{i}^{\min } \leq P_{i} \leq P_{i, 1}^{L B} \\
P_{i, j-1}^{U B} \leq P_{i} \leq P_{i, j}^{L B} \ldots j=2,3, \ldots, N P_{i} \\
P_{i, j}^{U B} \leq P_{i} \leq P_{i}^{\max } \ldots j=N P_{i}
\end{array}\right\}
$$

For $\mathrm{i}=1,2, \ldots \mathrm{N}$. The unit $\mathrm{i}$ having NPi unauthorized zones can have $\mathrm{NP}_{\mathrm{i}+1}$ disconnected operating conditions; generates a non-convex set.

\section{DIFFERENTIAL EVOLUTION}

Storn and Price designed a Differential Evolution (DE) which is one of the algorithm with regularities in optimization. The operators of DE like crossover, mutation and selection is as same as other population based algorithm. When compared to genetic algorithm which based on crossover DE depends upon mutation operators for better optimization. the performance and its working depending upon difference among a sampled pair of solution chosen randomly in population.

Differential Evolution (DE) undertakes NP D-dimensional seek variable vectors namely "chromosomes" in its parallel direct search method. The successive generation is given by discrete steps like $t=0,1,2, \ldots, t, t+1$, etc. In different generations the vectors are liable to transformation, so the $\mathrm{i}^{\text {th }}$ vector represents population at current generator can be written as

$$
\vec{X}_{i}(t)=\left[x_{i, 1}(t), x_{i, 2}(t), x_{i, 3}(t) \ldots x_{i, D}(t)\right]
$$

For better search results, there might be certain level for a variable seek within which value become false. At the commencement of time $(t=0)$ in DE process, initialization of independent variables or problem parameters at somewhere in their viable mathematical range. The $\mathrm{j}^{\text {th }}$ term in population can be specified as:

$$
x_{i, j}(0)=x_{j}^{L}+\operatorname{rand}(0,1) \cdot\left(x_{j}^{U}-x_{j}^{L}\right)
$$


Solving Wind Integrated Dynamic Economic Load Dispatch using Dynamic Neighborhood based Differential Evolution

Where $\mathrm{x}^{\mathrm{L}}$ and $\mathrm{x}^{\mathrm{U}}$ is the minor and major bound of the $\mathrm{j}^{\text {th }}$ parameter and rand $(0,1)$ is a consistently scattered arbitrary number lies within 0 and 1 .

The following defines rule involved in DE : Initialization, Mutation, Crossover and Selection.

\subsection{Initialization}

Initial Population $\mathrm{P}_{0}$ is produced in this step which contains $\mathrm{N}_{\mathrm{P}}$ individuals $\mathrm{x}^{0}, \mathrm{i}, 1 \leq \mathrm{i} \leq \mathrm{N}_{\mathrm{P}}$

$$
X_{j}^{0, i}=b_{j}^{L}+\alpha_{j}^{i}\left(b_{j}^{U}-b_{j}^{L}\right) \quad 1 \leq j \leq N
$$
number

Where $\left(b_{j}^{U}-b_{j}^{L}\right)$ is the boundary of the $\mathrm{j}^{\text {th }}$ optimization bound; $\alpha_{j}{ }^{i}$ denoted as random

Also not obligatory consistent in range $[0,1]$

\subsection{Mutation}

After initialization, through mutation and sometimes through arithmetic recombination donor vector $V_{i, j} \rightarrow$ gets created in production of current in relation with each population member $X_{i, G} \rightarrow \quad \mathrm{F}$ is a scaling factor, which denotes a differential vector scaling oriented with positive controllable parameter. $X_{\text {best }, G}$ denotes a vector which having stability in population generation. From above, differentiating DE from others by donor vector. For producing donor vectors, the most important strategies in DE are arranged below.

$$
\begin{aligned}
& \text { "DE/rand } / 1 ": \vec{V}_{i, G}=\vec{X}_{r_{1}^{i}, G}+F \cdot\left(\vec{X}_{r_{2}^{i}, G}-\vec{X}_{r_{3}^{i}, G}\right) \\
& \text { "DE/best/1": } \vec{V}_{i_{3}, G}=\vec{X}_{\text {best }, G}+F \cdot\left(\vec{X}_{r_{1}^{i}, G}-\vec{X}_{r_{2}^{i}, G}\right) \\
& \text { "DE/t } \arg \text { et -to-best } / 1 ": \vec{V}_{i, G}=\vec{X}_{i, G}+F \cdot\left(\vec{X}_{\text {best }, G}-\vec{X}_{i, G}\right)+F \cdot\left(\vec{X}_{r_{1}^{i}, G}-\vec{X}_{r_{2}^{i}, G}\right) \\
& \text { "DE / best / 2": } \vec{V}_{i, G}=\vec{X}_{\text {best }, G^{\prime}}+F \cdot\left(\vec{X}_{r_{1}^{i}, G}-\vec{X}_{r_{2}^{i}, G}\right)+F \cdot\left(\vec{X}_{r_{3}^{i}, G}-\vec{X}_{r_{4}^{i}, G}\right) \\
& \text { "DE/rand } / 2 ": \vec{V}_{i, G}=\vec{X}_{r_{1}^{i}, G}+F \cdot\left(\vec{X}_{r_{2}^{i}, G}-\vec{X}_{r_{3}^{i}, G}\right)+F \cdot\left(\vec{X}_{r_{4}^{i}, G}-\vec{X}_{r_{5}^{i}, G}\right)
\end{aligned}
$$

Where $\mathrm{r}_{1}{ }^{\mathrm{i}}, \mathrm{r}_{2}{ }^{\mathrm{i}}, \mathrm{r}_{3}{ }^{\mathrm{i}}, \mathrm{r}_{4}{ }^{\mathrm{i}}, \mathrm{r}_{5}{ }^{\mathrm{i}}$ are the vectors belonging to randomly chosen from [1, NP]; differ from base index i. DE/x/y/z is used to name various mutation strategies, where DE represents the Differential evolution, $\mathrm{x}$ stands for a string tends to perturbed vector, $\mathrm{y}$ represents $\mathrm{N}$ vectors taken for perturbing $\mathrm{x}$, and $\mathrm{z}$ represents crossover.

\subsection{Crossover}

The crossover scheme in DE algorithms is further classified into exponential and binomial.

The trial vector is formed by exchanging certain things in donor with target vector,

$$
\vec{U}_{i, G}=\left[u_{1, i, G}, u_{2, i, G}, \ldots, u_{D_{2}, i_{2} G}\right]
$$

The exponential crossover can be outlined by equation which is given below

$U_{j, i, G}=\left\{\begin{array}{c}v_{j, i, G} \text { for } j=\langle n\rangle_{D},\langle n+1\rangle_{D}, \ldots,\langle n+L-1\rangle_{D} \\ x_{j, i, G} \text { for all other } j \in[1, D]\end{array}\right.$ 
The binomial crossover evolved within it is outlined by following equation

$U_{j, i, G}=\left\{\begin{array}{c}v_{j, i, G} \text { if }\left(\text { rand }_{i, j}(0,1) \leq C r \text { or } j=j_{\text {rand }}\right) \\ x_{j, i, G} \text { otherwise }\end{array}\right.$

\subsection{Selection}

The operation can be demonstrated as

$$
\begin{aligned}
\vec{X}_{i, G+1} & =\vec{U}_{i, G}, \text { if } f\left(\vec{U}_{i, G}\right) \leq f\left(\vec{X}_{i, G}\right) \\
& =\vec{X}_{i, G} \text { if } f\left(\vec{U}_{i, G}\right)>f\left(\vec{X}_{i, G}\right)
\end{aligned}
$$

Where $\mathrm{f}\left(\mathrm{X}_{\mathrm{i}, \mathrm{G}}\right)$ has been minified.

\subsection{DEGL}

To avoid unnecessity of the classical DE, neighborhood topology based a new DE- variant was developed in the year 2006. The proposed methodology includes proposed a neighboring local mutation that brings attention from PSO. For each population member, by employing the fittest and two other vectors from same neighborhood results in the creation of local mutation.

The model can be written by,

$$
\vec{L}_{i, G}=\vec{X}_{i, G}+\alpha \cdot\left(\vec{X}_{n_{-} \text {best }, G}-\vec{X}_{i, G}\right)+\beta \cdot\left(\vec{X}_{p, G}-\vec{X}_{q, G}\right)
$$

Where vector $\vec{X}_{n_{-} b e s t, G}$ is excellent vector in neighbourhood of $\vec{X}_{i, G}$ and p, q $\epsilon$ [i-k, i $\left.+\mathrm{k}\right]$ including $\mathrm{p} \neq \mathrm{q} \neq \mathrm{i}$. The global donor vector can be represented by

$$
\vec{g}_{i, G}=\vec{X}_{i, G}+\alpha \cdot\left(\vec{X}_{g_{-} \text {best }, G}-\vec{X}_{i, G}\right)+\beta \cdot\left(\vec{X}_{r 1, G}-\vec{X}_{r 2, G}\right)
$$

Where,

$\alpha$ and $\beta$-scaling factors

$\vec{X}_{g_{-} b e s t, G}$ - Best vector within G \& r1, r2 $\epsilon[1, \mathrm{NP}]$ with $\mathrm{r} 1 \neq \mathrm{r} 2 \neq \mathrm{i}$.

The donor vector of DEGL is formed by combining the local and global donor vector use scalar weight $\mathrm{w} \in(0,1)$

$$
\vec{V}_{i, G}=w \cdot \dot{g}_{i, G}+(1-w) \cdot \vec{L}_{i, G}
$$

The conventional DE is resembled in the rest of the algorithm,

Neighborhood vector is the set of interconnected other parameter vector's and it updates the position considering previous experience, whose graph is named as neighborhood structure. In regional model, the parameter vector identifies an excellent area on search space its immediate neighbors only get influenced directly. Until they become highly successful its second degree neighbors doesn't begin. The inconvenience in spreading information through population reveals the platform of neighborhood. The prevention of population from getting trapped in local minima was achieved through weakened attraction of specific points.

\section{SIMULATION RESULTS}

The outcome of the model is analyzed through two case studies. Each case is studied under simulation having 50 trials to understand robustness of proposed method by comparing it with other optimizing techniques. The required components in addition with objective function of 
Solving Wind Integrated Dynamic Economic Load Dispatch using Dynamic Neighborhood based Differential Evolution

fuel cost are validated by simulating valve point effect. The optimization process including generators, fuel cost, losses consideration, etc. the simulation study is performed in MATLAB-R2014 with 32 bit. In proposed system $\mathrm{N}$ tends to be 50, $\mathrm{F}$ becomes 0.8 ; $\mathrm{G}_{\max }=500$.

\subsection{Case Study 1}

The record about 15 unit systems data further retrieves data about valve point loading, variation among $\mathrm{P}_{\min }$ and $\mathrm{P}_{\max }$, fuel cost estimation, ramp rate limit, unauthorized operating zones, power requirement of $2630 \mathrm{MW}$ and need of wind energy for a day in [16]. The data present in table 1 visualizes the results; describes frequency of convergence under 50 trial runs. The vast methodology reviewed through literature survey also explains about the essentialities and the outcome of proposed system [17].

Table 1 Experimental Results (50 Trials)

\begin{tabular}{|l|c|c|c|}
\hline \multirow{2}{*}{\multicolumn{1}{|c|}{ Method }} & \multicolumn{3}{|c|}{ Total Generation Cost (\$) } \\
\cline { 2 - 4 } DE / Best / 1 & Minimum Cost & Mean Cost & Maximum Cost \\
\hline $\mathrm{DE} /$ Best / 2 & 32913.456 & 32949.299 & 32985.143 \\
\hline $\mathrm{DE} /$ Rand / 1 & 32548.249 & 32595.398 & 32642.547 \\
\hline $\mathrm{DE} /$ Rand / 2 & 32665.376 & 32686.766 & 32708.156 \\
\hline $\mathrm{DEGL}$ & 32548.289 & 32578.351 & 32608.413 \\
\hline
\end{tabular}

Table 2 Frequency of Convergence (50 Trials)

\begin{tabular}{|c|c|c|c|c|c|}
\hline \multirow{2}{*}{ Method } & \multicolumn{5}{|c|}{ Cost variation (\$) } \\
\cline { 2 - 6 } & $32450-32500$ & $32500-32550$ & $32550-32600$ & $32600-32650$ & $32650-32700$ \\
\hline DEGL & 0 & 20 & 18 & 12 & 0 \\
\hline
\end{tabular}

\subsection{Case Study 2}

The record study about 10 unit systems data further retrieves data about valve point loading, fuel cost estimation, ramp rate limit, unauthorized operating zone, power requirement; need of wind energy for a day in [18].

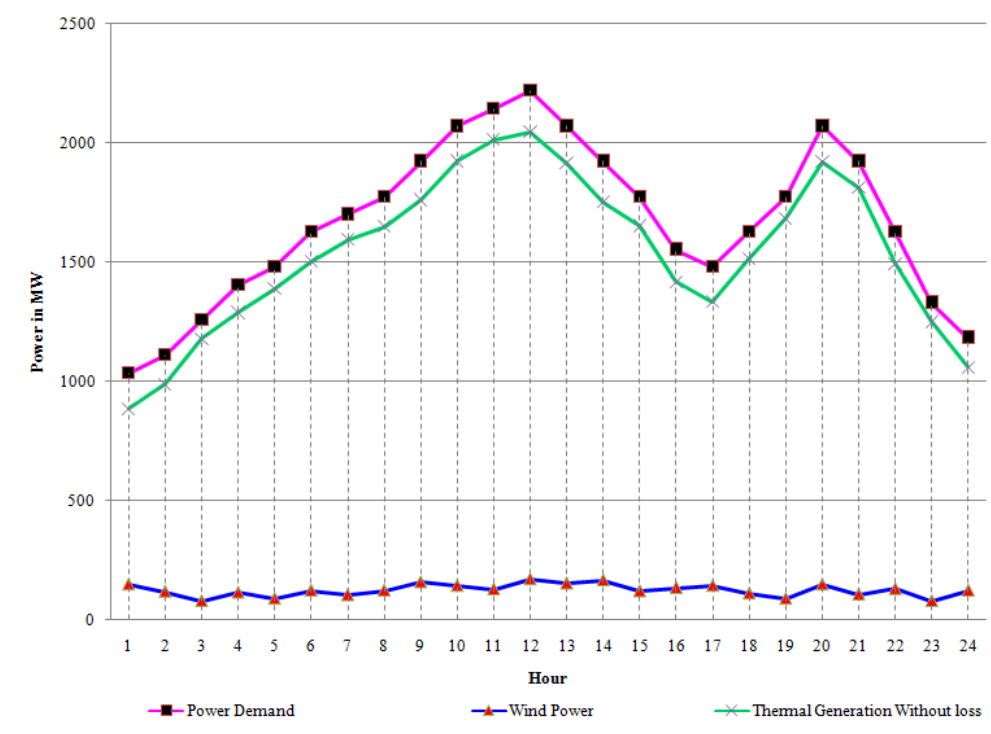

Figure 1 Daily Load curve 
In Figure 1, it was presented the daily load curve subjected to the 10 unit system with the support of variable wind power generation [21]. Also it was clearly stated about the amount of thermal power generation required for the demand to get satisfied.

Table 3 Convergence of Fuel Cost (10 Unit Systems)

\begin{tabular}{|c|c|c|c|c|c|c|}
\hline Hour & $\mathbf{1}$ & $\mathbf{2}$ & $\mathbf{3}$ & $\mathbf{4}$ & $\mathbf{5}$ & $\mathbf{6}$ \\
\hline $\begin{array}{c}\text { FC } \\
(\$)\end{array}$ & 52991.131 & 56625.029 & 68043.199 & 72439.656 & 77821.669 & 85788.52 \\
\hline Hour & 7 & 8 & 9 & 10 & 11 & 12 \\
\hline $\begin{array}{c}\text { FC } \\
(\$)\end{array}$ & 90207.777 & 93465.344 & 101285.578 & 117473.592 & 126412.908 & 129788.284 \\
\hline Hour & 13 & 14 & 15 & 16 & 17 & 18 \\
\hline $\begin{array}{c}\text { FC } \\
(\$)\end{array}$ & 115183.48 & 101007.778 & 94620.632 & 79653.555 & 75257.424 & 85282.967 \\
\hline Hour & 19 & 20 & 21 & 22 & 23 & 24 \\
\hline $\begin{array}{c}\text { FC } \\
(\$)\end{array}$ & 95683.969 & 115762.382 & 105340.121 & 84604.617 & 69864.341 & 60279.781 \\
\hline
\end{tabular}

\section{CONCLUSION}

Thus progress of proposed load dispatch having valve point effect differential evolution algorithm associated with neighborhood scrutinize operator is described in the paper. Factors such as probability factor, replacement size, and mutation rate have been designed through proposed algorithm to attain solution for the test case enrolled with it. The case study on 13 units, 10 unit systems revealed the valve-point effect, ramp rate limit and then obtains a comparative knowledge about other methodologies. The priority of proposed optimization technique over other methods is hence proven by solutions quality, convergence property, strength at test occurs in each case.

\section{REFERENCES}

[1] P. Attaviriyanupap, H. Kita, E. Tanaka, J. Hasegawa, (2002) A hybrid EP and SQP for dynamic economic dispatch with nonsmooth fuel cost function, IEEE Trans. Power Syst. 17 (May (2)), 411-416.

[2] A.H. Rashed, E.N. Abdallah, F. Benhamida, (2005,) A hopfield neural network based dynamic dispatch solver, in: Proceedings of the 10th International Middle East Power System Conference, MEPCON'05, December, pp. 515-525.

[3] T.A.A. Victoire, A.E. Jeyakumar, (2005) Reserve constrained dynamic dispatch of units with valve-point effects, IEEE Trans. Power Syst. 20 (August (3)) 1272-1282.

[4] B.Padmanabhan, L.Premalatha, (2019)“'Adaptive Bacterial Foraging Algorithm to Green Energy Supported Non Smooth Dynamic Power Dispatch Optimization” Journal of Green Engineering, Vol.9 (3), October, PP. $402-410$.

[5] M. Basu, (2011) Artificial immune system for dynamic economic dispatch, Electric power and energy systems, 33, 131-136.

[6] R. Storn, K. Price, (1997) "Differential Evolution - A simple and efficient adaptive scheme for global optimization over continuous spaces,"[Online]. Journal of Global Optimization, vol. 11, Dordrecht, pp. 341-359.

[7] Varadarajan, M., Swarup, K., (2008). Differential evolutionary algorithm for optimal reactive power dispatch. International Journal of Electrical Power and Energy Systems 30 (8), 435-441.

[8] Das S, Abraham A, Chakraborty UK, Konar A. (2009) Differential evolution using a neighborhood-based mutation operator. IEEE Trans Evol Comput; 13(3):526-53. 
Solving Wind Integrated Dynamic Economic Load Dispatch using Dynamic Neighborhood based Differential Evolution

[9] Noman N, Iba H. (2008) Accelerating differential evolution using an adaptive local search. IEEE Trans Evol Comput; 12(1):107-25.

[10] Lampinen J, Zelinka I. (2000) On stagnation of the differential evolution algorithm. In: Osmera P, editor. Proceedings of $6^{\text {th }}$ International Mendel Conference on Soft Computing; p. $76-83$.

[11] Liu J, Lampinen J. (2002) On setting the control parameter of the differential evolution algorithm. In: Matousek R, Osmera P, editors. Proceedings of the 8th International Mendel Conference on Soft Computing, p. 11-8.

[12] B.Padmanabhan, L.Premalatha, "Honey Bees Searching Optimization for Non Convex Dynamic Power Dispatch Problem in Thermal Units", Indian Journal of Science and Technology, Vol 8(18), August 2015, pp. 1-6.

[13] Z.X. Liang and J.D. Glover, (1992) "Zoom feature for a programming solution to economic dispatch including transmission losses", IEEE Trans. Power Syst., vol.7, no. 3, pp. 554- 550.

[14] B.Padmanabhan, J.Jasper, Sivakumar R.S., (2011) "Bee Hive Algorithm to Optimize Multi Constrained Piecewise Non-Linear Economic Power Dispatch Problem in Thermal Units" International Journal on Electrical Engineering and Informatics - Vol. 3, No. 1, pp. $109-117$

[15] Swagatam Das, Ajith Abraham, K. Chakraborty, and Amit Konar, (2009) Differential Evolution Using a Neighborhood-Based Mutation Operator, IEEE Trans. Evol. Comp. 13(3) 526-553.

[16] K.T. Chaturvedi, Manjareepandit, Laxmi Srivastava, (2008) "Self-Organizing Hierarchical Particle swarm optimization for non convex Economic Dispatch," IEEE Trans. Power Syst., 23(1), 1079-1087.

[17] B.Padmanabhan, Sivakumar R.S, J.Jasper, (2011) "DEGL based optimization for practical constrained Economic Power Dispatch problem" Journal of Electrical Engineering: Volume 11 / 2011 - Edition: 3, pp. 26 - 32.

[18] J.P.Chiou, (2009) "A variable scaling hybrid differential evolution for solving large-scale power dispatch problems", IET Generation, Transmission \& Distribution, Vol. 3, Issue 2, pp. $154-163$.

[19] B. Padmanabhan, R.S. Sivakumar, J. Jasper, T. Aruldoss Albert Victoire, (2011) "Bacterial Foraging Approach to Economic Load Dispatch Problem with Non Convex Cost Function", SEMCCO 2011, Part I, LNCS 7076, Springer-Verlag Berlin Heidelberg, pp. 577-584.

[20] Tao Ding, Zhaohong Bie, (2017) "Parallel Augmented Lagrangian Relaxation for Dynamic Economic Dispatch Using Diagonal Quadratic Approximation Method" IEEE Transactions on Power Systems, Vol. 32, No. 2, pp. 1115-1126.

[21] Padmanabhan, B., Premalatha, L. (2019) "A statistical analysis in optimization of wind penetrated non convex dynamic power dispatch problem using different strategies of differential evolution algorithm". J Ambient Intell Human Comput. https://doi.org/10.1007/s12652-019-01562-1

[22] Vijay Kumar, Jagdev Singh, Yaduvir Singh and Sanjay Sood. (2016) Optimal Economic Load Dispatch Using Fuzzy Logic \& Genetic Algorithms. International Journal of Computer Engineering and Technology, 7(1), pp. 62-77.

[23] Vijay Kumar, Dr. Jagdev Singh, Dr. Yaduvir Singh, Dr. Sanjay Sood, (2012) Design \& Development of Genetic Algorithms for Economic Load Dispatch of Thermal Generating Units, International Journal of Computer Engineering and Technology, 3(1), pp. 59-75. 